\title{
História Familiar em Segundo Grau como Fator de Risco para Câncer de Mama
}

\author{
Second-Degree Family History as
}

a Risk Factor for Breast Cancer

Rafael Marques de Souza, Anderson Rech Lazzaron, Rafael Defferrari Álvaro A. Borba, Luciana Scherer, Antônio L. Frasson

\section{RESUM0}

\begin{abstract}
Objetivos: investigar a associação entre história familiar de câncer de mama em segundo grau e o risco de apresentar a doença.

Métodos: estudo de caso-controle com casos incidentes. Foram avaliados 66 casos e 198 controles selecionados entre mulheres que realizaram mamografia em Serviço Privado de Radiodiagnóstico no período de janeiro de 94 a julho de 97. Casos e controles foram pareados quanto idade, idade da menarca, da primeira gestação e da menopausa, paridade, uso de anticoncepcionais orais e terapia de reposição hormonal.

Resultados: não houve diferença significativa entre casos e controles em relação a outros fatores de risco que não história familiar em segundo grau. As pacientes com câncer de mama apresentaram maior chance de ter história familiar em segundo grau comparadas aos controles (RC=2,77; IC 95\%, 1,03-7,38; $p=0,039$ ).

Conclusões: a neoplasia maligna de mama está associada à presença de história familiar em segundo grau para essa doença.
\end{abstract}

PALAVRAS-CHAVE: Mama: câncer. Câncer: epidemiologia. Fatores de risco. Hereditariedade.

\section{Introdução}

O câncer de mama é um problema de saúde pública importante. Estima-se que cerca de 12,2\% das mulheres americanas terão câncer de mama durante a vida. Dessas, 3,5\% irão morrer vítimas da doença ${ }^{11,14}$. A incidência de câncer de mama tem aumentado nas últimas décadas. Esse

Centro de Ecografia e Radiologia - Hospital São Rafael - Novo Hamburgo - RS. Faculdade de Medicina - Universidade Federal do Rio Grande do Sul. Faculdade de Medicina - Pontifícia Universidade Católica de Porto Alegre - RS

Correspondência:

Rafael Marques de Souza

Rua Heller 61/81

CEP 93510-330 - Novo Hamburgo/RS - Fax: (051) 5932032

e-mail: souza@nh.conex.com.br aumento não se explica apenas pelo implemento dos programas de rastreamento mamográfico, mas também por uma elevação real ${ }^{5,7}$.

Nas últimas décadas, vários estudos têm identificado fatores de risco associados ao câncer de mama, sendo a grande maioria retrospectivos a história familiar de câncer de mama, principalmente em primeiro grau, tem sido associada a um aumento no risco de desenvolver esta doença. Estudos prévios sugerem que o risco é maior em mulheres com história familiar de câncer de mama na pré-menopausa (principalmente antes dos 40 anos de idade) e naquelas com história familiar de doença bilateral ${ }^{2,3,4,18}$. Outros estudos indicam que a história familiar não representa fator de risco para câncer de mama em mulheres com mais de $60 \operatorname{anos}^{10,15}$, ao passo que 
há afirmativas de que o risco nesta faixa etária também é elevado, porém de menor magnitude ${ }^{16}$. Acredita-se que mulheres cuja tia ou avó apresentaram câncer de mama têm 1-2 vezes mais chance de desenvolvê-1o ${ }^{13,17}$. No entanto, apenas 10\% das pacientes acometidas apresentam história familiar9. Alguns pesquisadores, entretanto, acreditam que tal magnitude seja superestimada em razão de viéses de seleção e de aferição do tipo recordativo, comuns em estudos de caso-controle ${ }^{6,8}$. Isso ocorreria porque as pacientes com câncer de mama estariam mais informadas a respeito de sua história familiar e poderiam relatar mais acuradamente, ao invés de pacientes sem a doença, que não estão ao par dos casos em sua família. Estudos prospectivos não são sujeitos a estes tipos de viéses.

Conduzimos um estudo de caso-controle contemporâneo para avaliar a associação entre história familiar de câncer de mama em segundo grau e o risco subseqüente de apresentar a mesma doença em mulheres submetidas à mamografia em serviço privado de radiodiagnóstico.

\section{Material e Métodos}

Utilizamos como delineamento um estudo de caso-controle, no qual se utilizou casos incidentes para determinar o risco de apresentar história familiar em segundo grau para câncer de mama em mulheres portadoras dessa doença.

Mulheres submetidas a mamografia em serviço privado de radiodiagnóstico no Vale dos Sinos/RS, no período de janeiro de 94 a julho de 97 foram entrevistadas previamente à realização do exame, respondendo questionário padronizado sobre os seguintes fatores de risco para câncer de mama: idade da menarca e menopausa (caso houvesse ocorrido), idade da primeira gestação (caso houvesse ocorrido), paridade, amamentação, uso atual (por pelo menos 30 dias) de anticoncepcionais orais (ACO) ou terapia de reposição hormonal (TRH), além de história familiar em primeiro ou segundo graus para câncer de mama. Foi considerada como história familiar de câncer de mama em primeiro grau a existência de mãe ou irmã com diagnóstico prévio da doença, referida pela paciente. Mulheres que referiram ter avó ou tia com diagnóstico prévio da doença foram consideradas como tendo história familiar em segundo grau. As mulheres foram consideradas pós-menopáusicas caso estivessem há mais de 12 meses em amenorréia no momento da aplicação do questionário. As mulheres que referiram histerectomia sem ooforectomia bilateral foram consideradas pós-menopáusicas quando sua idade no momento da aplicação do questionário era igual ou superior ao percentil 90 da ocorrência de menopausa na população estudada. Foram excluídas do estudo todas as mulheres que apresentavam história familiar de câncer de mama em primeiro grau, limitando dessa forma o potencial de confusão exercido por esse fator.

Após o exame, todas as pacientes que apresentavam lesões suspeitas de malignidade segundo avaliação do mamografista (pelo exame físico, mamografia e ecografia quando necessária), foram acompanhadas em sua investigação complementar até que se obtivesse o diagnóstico anatomopatológico da lesão observada no exame. Consideramos portadoras de neoplasia maligna primária de mama todas as pacientes que obtiveram confirmação histológica, sendo incluídas tanto lesões in situ como invasoras. Tais pacientes constituíram a população de casos. Do restante das pacientes, provenientes da mesma região geográfica e que realizaram mamografia no mesmo serviço e período sem ter apresentado diagnóstico de câncer de mama, foram selecionados três controles por caso. Casos e controles foram pareados para idade ( \pm 2 anos), idade da menarca ( \pm 2 anos), idade da menopausa ( \pm 3 anos), idade da primeira gestação ( \pm 3 anos), paridade (nulíparas ou não-nulíparas), status menopáusico (pré ou pósmenopáusicas), uso de ACO e TRH. Nem casos nem controles apresentavam história pessoal prévia de neoplasia maligna de mama.

O protocolo foi aprovado pelo Comitê de Ética da Instituição onde foi realizado o estudo. As pacientes foram informadas da natureza do estudo, consentindo em participar.

Após a seleção de casos e controles, os dois grupos foram comparados quanto a fatores de risco que poderiam influenciar a medida de associação entre câncer de mama e história familiar da doença em segundo grau. Comparou-se idade, idade da menarca, idade da menopausa, idade da primeira gestação, paridade, uso de anticoncepcionais orais e terapia de reposição hormonal. Para fatores de risco descritos por variáveis contínuas (idade, idade da menarca, idade da menopausa, idade da primeira gestação) utilizou-se o teste t de Student para comparação da homogeneidade dos grupos. As tabelas de freqüência foram analisadas pelo teste $\chi^{2}$ (distribuição normal/paramétrico-Yates corrigido) ou Mann-Whitney (distribuição nãonormal/não-paramétrico). Utilizamos a razão de chances como medida de associação. Os grupos foram considerados homogêneos para os fatores de risco quando o teste estatístico utilizado apresentou $\mathrm{p} \geq 0,05$. 


\section{Resultados}

A população consistiu de 66 casos e 198 controles. Não houve diferença estatisticamente significativa entre casos e controles em relação a idade, idade da menarca, da menopausa, status menopáusico, idade da primeira gestação, paridade, uso de anticoncepcionais orais e terapia de reposição hormonal (Tabela 1).

Tabela 1 - Características de casos e controles quanto aos fatores de risco para câncer de mama.

\begin{tabular}{lccr}
\hline Característica & & & \\
& Casos (n=66) & Controles (n=198) & p \\
\hline Idade (anos) & $54,5 \pm 12,9$ & $54,1 \pm 12,5$ & 0,84 \\
Idade da menarca (anos) & $13,1 \pm 1,8$ & $13,2 \pm 1,5$ & 0,72 \\
Nulíparas (\%) & 16,7 & 13,6 & 0,54 \\
Idade da $1^{\circ}$ gestação (anos) & $23,0 \pm 4,3$ & $22,9 \pm 4,0$ & 0,90 \\
$\mathrm{~N}^{\circ}$ de filhos & $2,3 \pm 1,9$ & $2,4 \pm 1,8$ & 0,55 \\
Pré-menopáusicas (\%) & 45,5 & 44,9 & 0,94 \\
Idade da menopausa (anos) & $50,4 \pm 5,0$ & $50,5 \pm 4,3$ & 0,91 \\
Uso de ACO (\%) & 6,1 & 8,1 & 0,59 \\
Uso de TRH (\%) & 12,1 & 15,8 & 0,48 \\
& & & \\
\hline
\end{tabular}

* Valores estão expressos em média \pm desvio padrão, a menos que indicado de outra forma.

As pacientes com câncer de mama apresentaram maior chance de ter história familiar em segundo grau para a doença quando comparadas aos controles $(15,15 \%$ versus $6,06 \%$; $\mathrm{RC}=2,77$; IC 95\% , 1,03-7,38; $\mathrm{p}=0$,039) (Tabela 2).

Tabela 2 - Associação entre história familiar de câncer de mama em segundo grau e risco de câncer de mama.

\begin{tabular}{lcccc} 
& $\begin{array}{c}\text { Casos } \\
(\mathrm{n}=66)\end{array}$ & $\begin{array}{c}\text { Controles } \\
(\mathrm{n}=198)\end{array}$ & $\mathrm{RC}$ & $\begin{array}{c}\text { IC } \\
95 \%\end{array}$ \\
\hline $\begin{array}{l}\text { Mulheres sem história } \\
\text { familiar de câncer de } \\
\text { mama em segundo grau }\end{array}$ & 56 & 186 & 1,00 & - \\
$\begin{array}{l}\text { Mulheres com tia ou avó } \\
\text { com história }\end{array}$ & 10 & 12 & 2,77 & $1,03-7,38$ \\
de câncer de mama & & & & \\
\hline
\end{tabular}

\section{Discussão}

Neste estudo, observamos uma associação significativa entre história familiar de câncer de mama em tia ou avó e diagnóstico dessa doença em mulheres submetidas à mamografia. Nossos achados concordam com os de estudos prévios, estabelecendo uma associação significativa entre história familiar de segundo grau e câncer de mama $^{13,17}$. A magnitude de associação encontrada $(\mathrm{RC}=2,77)$ foi um pouco superior à relatada em estudos prévios, o quais encontraram uma razão de chances entre $1,50^{13}$ e $1,82^{17}$.

Como havia disponibilidade de dados referentes a um grande número de pacientes sem a doença (2.597 pacientes), ao invés de obter-se uma amostra aleatória da população para representar os controles (avaliando a razão de chances, posteriormente, por meio de análise estratificada), selecionou-se um grupo de pacientes com as mesmas características dos casos (pareadas individualmente na proporção 3:1) no que se referia aos potenciais fatores de confusão dos resultados. Dessa forma, constituíram-se duas populações (casos e controles) homogêneas em relação a idade, idade da menarca, paridade, idade da primeira gestação, status menopáusico, idade da menopausa, uso de ACO e TRH. Isolou-se, dessa forma, o fator de risco em estudo para que se obtivesse uma avaliação mais acurada da associação.

A relação entre história familiar em segundo grau e câncer de mama não foi afetada pelo status menopáusico ou idade da paciente. Apesar de a população estudada ser predominantemente composta por mulheres brancas, dados de outros estudos sugerem que a associação entre história familiar e câncer de mama é semelhante em mulheres negras ${ }^{1}$.

O principal problema associado à participação no estudo de pacientes provenientes de um serviço particular de mamografia é o fato de que os resultados podem ser afetados pela autoseleção, ou seja, mulheres que apresentam história familiar de câncer de mama apresentam maior probabilidade de realizar o exame mamográfico do que mulheres que não apresentam parentes com a doença. Isto produziria um aumento na proporção de pacientes com história familiar nos controles e desta forma diminuiria a razão de chances. Assim, a magnitude dos resultados por nós encontrados poderia ser, de certa forma, subestimada. Como em nosso estudo, considerando toda a população de 2.663 pacientes analisada inicialmente, a proporção de pacientes com história familiar para câncer de mama foi de $11,0 \%$, semelhante às proporções encontradas em grupos controles de outros estudos $\left(12,3 \%{ }^{12} ; 17,9 \%{ }^{16} ; 18,6 \%{ }^{15}\right)$ e à estimada para a população em geral $(10,0 \%)^{9}$, isso possivelmente não constitui um fator de confusão importante. 
Como o questionário foi aplicado antes da paciente ter conhecimento do diagnóstico definitivo, o potencial viés de aferição do tipo recordativo não foi relevante. Acredita-se que, em estudos retrospectivos, a avaliação da história familiar é relatada cerca de $50 \%$ mais por pacientes com câncer do que pelas irmãs gêmeas dessas pacientes sem a doença ${ }^{6}$. Além disso, como os entrevistadores também não sabiam do diagnóstico das pacientes, eliminou-se a possibilidade de viés de aferição (interviewer bias) decorrente da maneira pela qual a exposição foi aferida. Uma proporção significativamente maior de casos apresentava algum sinal ou sintoma (relatado pela paciente) por ocasião do exame $(18,2 \%$ dos casos contra $8,6 \%$ dos controles; $p=0,03$ ). Isto poderia constituir um viés de aferição, visto que estas pacientes poderiam relatar de forma mais acurada sua história familiar por temerem estar com a doença. Tal viés recordativo poderia ter produzido um excesso de relatos de história familiar no grupo de casos (overreporting), aumentando falsamente a razão de chances encontrada. Portanto, havia um cegamento parcial dos entrevistadores e das mulheres entrevistadas quanto à presença ou não de câncer de mama, reduzindo a possibilidade de viéses sistemáticos.

As taxas de realização de mamografia poderiam diferir entre as pacientes com ou sem história familiar, visto que aquelas com a percepção de risco aumentado para a doença poderiam ser submetidas mais freqüentemente a mamografias e ter diagnósticos mais precoces, enviesando os resultados do estudo. Essa possibilidade de confusão existe em nosso estudo, visto que $72,7 \%$ das mulheres com história familiar em segundo grau relataram mamografia prévia, contra 44,6\% das mulheres sem tal fator de risco $(\mathrm{p}<0,001)$. Entre as submetidas a mamografia, o número médio de exames prévios apresentou diferença significativa ( 1,8 versus 0,8 exames; $p=0,002$ ) Isto significa que as mulheres com história familiar de câncer de mama estavam expostas a mamografias mais freqüentes (um rastreamento mais intenso), que poderia levar a um número excessivo de casos entre as pacientes com história familiar. Portanto, existe a possibilidade de que haja um viés de seleção do tipo rastreamento excessivo (surveillance bias), o qual produziria uma superestimação da razão de chances encontrada nesse estudo.

Todas as informações contidas nas análises foram baseadas em dados relatados pelos casos e controles. Para grande parte desta população, os eventos questionados ocorreram muitos anos antes da entrevista. Entretanto, acredita-se que fatores reprodutivos tendem a ser relatados com grande acurácia neste contexto ${ }^{12}$.
Desses resultados, concluímos que a história familiar em segundo grau é um importante fator de risco em nossa população, podendo representar tanto um componente genético como um componente ambiental compartilhado. O que pode contribuir para um risco aumentado nestas pacientes é a interação entre genético e ambiental. Se realmente existe essa interação, o risco na população seria muito maior do que se analisássemos o risco isolado associado a cada componente. Embora o fato de apresentar história familiar de câncer de mama não seja um fator modificável, é possível que uma predisposição genética da doença, representada pela história familiar, seja alterada pela modificação de fatores ambientais ainda não completamente definidos.

\section{SUMMARY}

Purpose: to evaluate the association between second-degree family history of breast cancer and the risk to develop the disease.

Methods: case-control study of incident cases. Sixty-six incident breast cancer cases and 198 controls were selected among women who were submitted to mammography in a private clinic between January 1994 and July 1997. Cases and controls were paired regarding age, age at menarche, at first live birth, at menopause, parity, oral contraceptives and use of hormonal replacement therapy.

Results: there was no significant difference between cases and controls regarding all risk factors evaluated, besides seconddegree family history. Patients with breast cancer were more likely to have second-degree relatives with breast cancer when compared to controls $(O R=2.77 ; 95 \%$ CI, 1.03-7.38; $p=0.039$ ).

Conclusions: malignant neoplasm of the breast is significantly associated with a second-degree family history of this disease.

KEY WORDS: Breast neoplasms. Risk factors. Heredity.

\section{Referências}

1.Amos CI, Goldstein AM, Harris EL. Familiality of breast cancer and socioeconomic status in blacks. Cancer Res 1991; 51:1793-7.

2.Anderson DE. Some characteristics of familial breast cancer. Cancer 1971; 28:1500-4.

3.Anderson DE. Genetic study of breast cancer: identification of a high risk group. Cancer 1974; 34:1090-7. 
4.Anderson DE, Badzioch MD. Risk of familial breast cancer. Cancer 1985; 56:383-7.

5.Colditz GA. Epidemiology of breast cancer. Findings from the nurses' health study. Cancer 1993; 71 (suppl 4):1480-9.

6.Floderus B, Barlow L, Mack TM. Recall bias in subjective reports of familial cancer. Epidemiology 1990; 1:318-21.

7.Harris JR, Lippman ME, Veronesi U, Willett W. Breast cancer. N Engl J Med 1992; 327:319-28.

8.Love RR, Evans AM, Josten DM. The accuracy of patient reports of a family history of cancer. J Chronic Dis 1985; 38:289-93.

9.McTiernan A, Gilligan MA, Redmond C. Assessing individual risk for breast cancer: risky business. $\mathrm{J}$ Clin Epidemiol 1997; 50:547-56.

10.Metllin C, Croghan I, Natarajan N, Lane W. The association of age and familial risk in a case-control study of breast cancer. Am J Epidemiol 1990;131:973-83.

11.Muir C, Waterhouse J, Mack T, Powell J, Whelan S, editors. Cancer incidence in five continents. vol. 5 . Lyon, France: International Agency for Research on Cancer, 1987. IARC Scientific publications $n^{\circ} 88$.
12.Paganini-Hill A, Ross RK. Reliability of reccall of drug usage and other health-related information. Am J Epidemiol 1982; 116:114-22.

13.Pharoah PD, Day NE, Duffy S, Easton DF, Ponder BA. Family history and the risk of breast cancer: a systematic review and meta-analysis. Int $J$ Cancer 1997; 71:800-9.

14.Ries LAG, Kosary CL, Hankey BF. SEER Cancer Statistics Review, 1973-1994: tables and graphs. Bethesda, MD: National Cancer Institute; 1997. NIH Publication No. 97-2789.

15.Roseman DL, Straus AK, Shorey W. A positive family history of breast cancer. Does its effect diminish with age? Arch Intern Med 1990; 150:191-4.

16.Sattin RW, Rubin GL, Webster LA, Hulzo CM, Wingo PA, Org HW. Familiy history and the risk of breast cancer. JAMA 1985; 253:1908-13.

17.Slattery ML, Kerber RA. A comprehensive evaluation of family history and breast cancer risk - The Utah Population Database. JAMA 1993; 270:1563-8.

18.Vassilarou D, Tzonou A, Vassilaros S. Familial occurence of breast cancer in a population with itermediate disease rates: a study in Greece. Cancer J 1990; 3:244-7. 